\title{
Fluorescence Spectroscopy
}

National Cancer Institute

\section{Source}

National Cancer Institute. Fluorescence Spectroscopy. NCI Thesaurus. Code C17738.

Fluorescent spectroscopy or fluorometry is a type of electromagnetic spectroscopy used for analyzing fluorescent spectra. It involves using a beam of light, usually ultraviolet light, that excites the electrons in molecules of certain compounds and causes them to emit light of a lower energy, typically, but not necessarily, visible light. 